Mittheilung aus dem chemischen Institut der Universität Erlangen und dem Laboratorium für angewandte Chemie von E. Beckmann in Leipzig.

\title{
Ueber die Einwirkung
}

von Dichloressigsäure auf Anilin und Homologe;

von Gustav Heller.

[Zweite Mittheilung.]

(Eingelaufen am 1j. December 1907.)

In einer früheren Mittheilung ${ }^{1}$ ) ist dieser Gegenstand schon experimentell von mir behandelt worden und es sollen die beschriebenen Versuche durch die folgenden Beiträge ergänzt und erweitert werden.

Es wurde zunächst die auf Seite 257 erwähnte Nitrirung der Stilbendicarbonsäure und die darauf folgende Reduction wiederbolt.

Für die Bereituvg grösserer Mengen Stilbendicarbonsäure ist der sicherste Weg der, dass man das nach L. Chalanay und E. Knoevenagela ${ }^{2}$ ) unter der Einwirkung von Jod und Natrium auf Benzylcyauid gebildete Dicyanstilben, dessen glatte Verseifung nach den üblichen Methoden Schwierigkeiten bietet, im Rohre mit Eisessig und Salzsäure auf $185^{\circ}$ erhitzt. Das

1) Diese Annalen 332, 247 u. ff.

$\left.{ }^{2}\right)$ Ber. d. deutsch. chem. Ges. 25, 288. 
erhaltene Diphenylmaleïnsäureanhydrid wurde mit starker Salpetersäure nitrirt, ein Theil des amorphen Nitroproductes dann mit Permangauat oxydirt und dabei 0 -Nitrobenzoësäure in geringer Menge neben der p-Verbindung mit Bestimmtheit nachgewiesen. Die Reduction der nitrirten Stilbendicarbonsäure lieferte ein Gemisch von Säuren, darunter die p,p'-Diamidostilbendicarbonsäure (Seite 282), sowie eine gelbe Säure, welche in heissem Wasser leicht löslich war und deren Constitution wegen der geringen Menge nicht bestimmt werden konnte. Wahrscheinlich ist sie durch Nitrirung in Ortbostellung und weitere Oxydation entstanden. Dass kein einfaches Gemenge ron p- und o-Nitrostilbencarbonsäuren bei der Nitrirung sich bildet, geht aus den Resultaten der synthetiscben Versuche hervor. Beide Nitrosäuren krystallisiren sehr leicht und ihre Mischung bleibt nie amorph, wie dies bei dem Reimer'schen Nitrirungsproducte der Fall ist.

Von den synthetisch dargestellten Stilbendicarbonsäuren feblten noch die m-Verbindungen. Das zu ihrer Gewinnung nöthige m-Nitrobenzylcyanid ist zwar schon von Salkowsky ${ }^{3}$ ) durch fractionirte Krystallisation der bei der Nitrirung von Benzylcyanid neben der $p$-Verbindung in geringer Menge entstehenden beiden Isomeren erbalten worden, aber auf diesem Wege nach eigenen Versucben nicht mit Sicherheit gewinnbar.

Gabriel4) hat die Substanz durch Umsetzung ron m-Nitrobenzylchlorid mit Cyankalium in alkoholisch-wässriger Lösung erbalten, aber nur in öliger Form. Lässt man die Reaction sich bei nicht $z u$ hoher Temperatur vollziehen, so krystallisirt das Umsetzungsproduct nach einiger Zeit und es kann so mNitrobenzylcyanid nach der Abscheidung einer nebenher entstehenden Substanz in beliebiger Menge dargestellt werden. Die weitere Verarbeitung geschah in ganz analoger Weise, wie bei der p- und o-Verbindung. Indes konnte die freie Amido-

s) Ber. d. deutsch. chem. Ges. 17, 506.

4) Ber. d. deutsch. chem. Ges. 16, 2064. 
säure nicht durch Reduction erhalten werden und zwar aus dem Grunde, weil das m,m-Dinitrostilbondicarbonsäureanhydrid auffallend beständig gegen saure Reductionsmittel ist und davon kaum verändert wird. Eine glatte Reduction findet nur statt, wenn man die Verbindung zunächst durch wenig Alkali in die Säure überfübrt und diese dann mit Zinkstaub und Ammoniak erhitzt, wobei die Reaction auch nur bis zur Bildung der Azoxyverbindung fortschreitet, die sich auch in saurer Lösung nicht weiter reduciren lässt. Es gelingt indessen, das Amidocyanid durch mehrstündiges Kochen seiner alkoholischen Lösung mit Kali-Natronlösung zu verseifen und daraus die Amidosäure abzuscheiden, wobei dieselbe in das Anhydrid übergeht. Dasselbe ist schwer löslich, hat charakteristische Eigenschaften, ist orange gefärbt und mit den Säuren aus Dichloressigsäure und Anilin nicht identisch.

Die auf Seite 285 gegebene Darstellung der Reduction der 0,0-Dinitrostilbendicarbonsäure muss dahin ergänzt, beziehungsweise berichtigt werden, dass mit Zinnchlorür nur die Azoxyverhindung erhalten wird, während die 0,0-Diamidostilbendicarbonsäure, welche mit der Säure aus Dichloressigsäure und Anilin vermuthlich identisch ist, bisher nicht synthetisch-erhalten werden kounte.

Noch nicht untersucht war bisher das Verhalten der Dichloressigsäure gegen m-Toluidin. Der Versuch ergab, dass dasselbe in ähnlicher Weise einwirkt, wie Anilin. Es, entstanden unter verschiedenen Versuchsbedingungen, gleichgültig ob ein oder zwei Mol. Base zur Verwendung kamen, zwei Substanzen, von welchen die eine sich in verdünnten Säuren und Alkalien löst, die andere dagegen in beiden Agentien unlöslich ist. Erstere Verbindung ist gelb, letztere röthlich gefärbt.

Ihren ganzen physikalischen und chemischen Eigenschaften, speciell dem Verhalten beim Schmelzen, der Farbe, sowie ihrer Schwerlöslichkeit in organischen Lösungsmitteln nach sind die Verbindungen Homologe der Reactionsproducte von Dichloressigsäure und Anilin. Einen Unterschied davon zeigt nur das 
eine der m-Toluidinderivate durch seine Löslichkeit in verdünnten Säuren. Nun wurde aber an unreinen Gemischen der beiden Säuren aus Anilin die Beobachtung gemacht, dass die Verbindungen in diesem Zustande sich erheblich leichter in verdünnten Mineralsäuren lösen.

Somit dürfte die Annahme gerechtfertigt sein, dass bei der Wechselwirkung von Dichloressigsäure und m-Toluidin Diamidodimethylstilbendicarbonsäuren und deren Toluide entstehen. Die Reindarstellung der Verbindungen, welche wahrscheinlich auch Gemenge von isomeren Substanzen darstellen, war nicht möglich.

Da somit drei principiell verschiedene Reactionen festgestellt waren, nach denen die Dichloressigsäure mit aromatischen Basen reagiren kann, wurde die Umsetzung auch auf die sechs isomeren Xylidine ausgedehnt, in der Erwartung, dass bier noch eine neue Reaction eintreten würde. Diese Annahme erwies sich als richtig. Das p-Xylidin giebt in glatter Weise eine Verbindung, welche sich von der primär entstehenden Di-p-xylidoessigsäure in der Art ableitet, dass beide basische Reste sich umgelagert haben zu einer Amidocarbonsäure von der Formel HOOC-CH[ $\left[\mathrm{C}_{6} \mathrm{H}_{2}\left(\mathrm{CH}_{3}\right)_{2} \mathrm{NH}_{2}\right]_{2}$. Die gut charakterisirte Verbiudung kuppelte mit zwei Mol. $\beta$-Naphtol, so dass über ihre Zusammensetzung kein Zweifel herrschen kann.

Diese Thatsache liess es nun angezeigt erscheinen, die von Paul J. Meyer beschriebene Di-o-toluidoessigsäure näher zu untersuchen. Leider sind die Derivate nicht sebr gut charakterisirt und musste deshalb von einer eingehenden Untersuchung abgesehen werden. Sehr wabrscheinlich ist es aber, dass diese Säure nicht die angenommene Constitution besitzt, sondern halbseitig umgelagert ist, also die Formel:

besitzt.

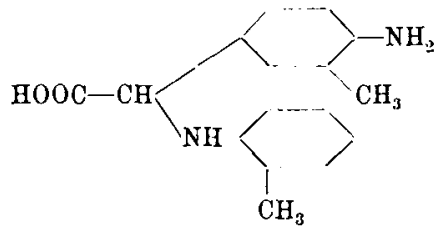


Ganz ähnliche Reactionen zeigt die Säure aus $m$-Xylidin-1,2,3; hier hinderte namentlich die schwere Löslichkeit der Derivate die genügende Charakterisirung.

Die gleiche Umsetzung wie p-Toluidin zeigten $\mathrm{m}$-Xylidin-1,3,4, m-Xylidin-1,3,5, 0-Xylidin-1,2,4, von denen die beiden ersten bezüglich ihrer Reactionsproducte mit Dichloressigsäure genauer untersucht wurden. Abweichend ist hier nur die grössere Beständigkeit des primär entstebenden Oxindolderivates und die in Folge dessen nothwendig werdenden anderen Oxydationsbedingungen. Im ersteren Falle lässt sich die Ueberführung in das Dimethylisatinxylidid mittelst Brom, im zweiten mit Hülfe von Jod durchführen; diese Substanzen werden dann aber gleich gespalten, so dass als Endproducte die Dimethylisatine selbst erscheinen.

Das o-Xylidin-1,2,3 schliesslich reagirt dem Anilin analog.

$\mathrm{Da}$ es, wie schon erwähnt ist, nicht möglich war, die Reactionsproducte der einzelnen Basen in allen Fällen genügend zu charakterisiren, so sind die betreffenden Versuche im experimentellen Theile nicht aufgeführt und sollen eventuell später näher beschrieben werden; dagegen liess sich die Art der Umsetzung in jedem Falle mit Sicherheit feststellen, wobei noch zu bemerken ist, dass die einzelnen Basen durchweg in dem jeweiligen Sinne einheitlich reagiren.

Die verschiedenen Umsetzungen können in folgende Klassen eingetheilt werden, wobei der Reactionsverlauf nach dem bisher vorliegenden Material in übersichtlicher Weise skizzirt ist.

I. $\mathrm{COOH}-\mathrm{CHCl}_{8}+2 \mathrm{H}_{2} \mathrm{~N}-\langle\cdots\rangle \mathrm{COOH}-\mathrm{CH}(\mathrm{HN}-\langle\rangle)_{2}$

Dieser einfacbste Fall ist bisher nicht beobachtet worden, es tritt vielmehr stets Umlagerung ein.

II.

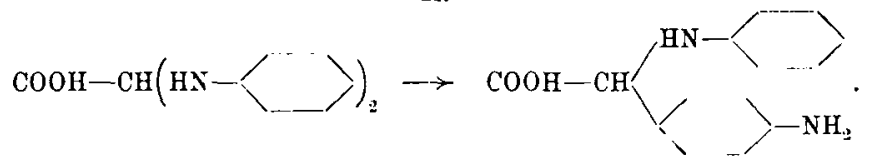


354 Heller, Ueber die Einwirkung von Dichloressigsäure

Die Reaction erfolgt wahrscheinlich bei o-Toluidin und m-Xylidin-1,2,3.

III.

$\mathrm{COOH}-\mathrm{CH}\left(\mathrm{HN}-\langle)_{2} \rightarrow \mathrm{COOH}-\mathrm{CH}\left(-\left\langle>-\mathrm{NH}_{2}\right)_{2}\right.\right.$.

Diese Umsetzung ist nachgewiesen für p-Xylidin.

IV.

$\mathrm{COOH}-\mathrm{CHCl}_{2}+\mathrm{H}_{2} \mathrm{~N}-\langle\rightarrow$

$\mathrm{COOH}-\mathrm{CHCl}-\mathrm{HN}-\langle\cdots \rightarrow \mathrm{COOH}-\mathrm{CH}=\mathrm{N}-\langle\cdots\rangle \rightarrow$<smiles>CCC1CCCC(C(C(=O)O)C(C(=O)O)C(C)N)C1N</smiles>

Dieser Verlauf ist sehr wahrscheinlich gemacht fur Anilin, m-Toluidin, o-Xylidin-1,2,3.

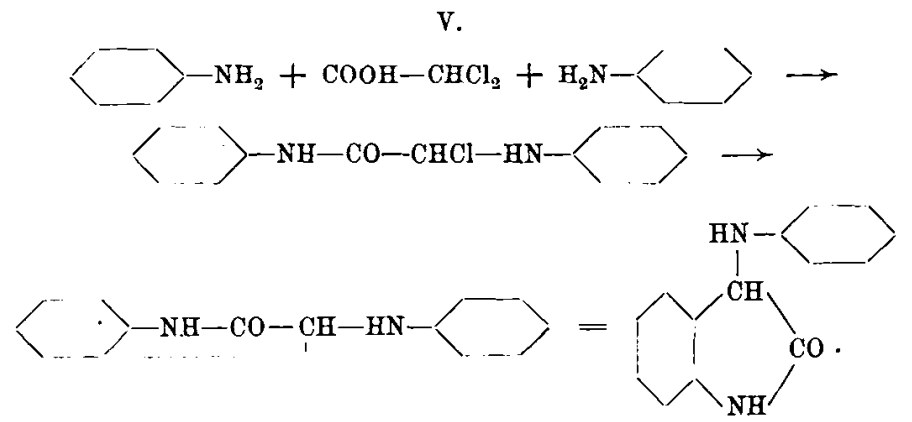

Nachgewiesen ist diese Bildung für p-Toluidin, m-Xylidin-1,3,4; m-Xylidin-1,3,5, o-Xylidin-1,2,4.

Es wird der Gegenstand weiterer Untersuchungen sein, die geschilderten Reactionen, soweit sie noch nicht vollständig sicher gestellt sind, zu vertiefen. 


\section{Experimenteller Theil.}

\section{Stilbendicarbonsäureanhydrid.}

Ein-Theil Dicyanstilben wurde mit zehn Theilen Eisessig und $1 \frac{1}{2}$ Theilen concentrirter Salzsäure fünf bis sechs Stunden auf $185^{\circ}$ erhitzt; der Röhreninhalt bildet nach dem Erkalten feine Nadeln neben grossen, würfelförmigen Krystallen, welche Stilbendicarbonsäure und ihr Amid sind. Dieselben wurden durch Kochen mit verdünnter Natronlauge in Lösung gebracht und aus der von etwas unverändertem Cyanid abfiltrirten Lösung durch Säuren Diphenylmaleïnsäureanhydrid abgeschieden, welches aus Alkohol und Wasser umkrystallisirt, den Schmelzpunkt $157^{\circ}$ zeigte.

\section{Nitrirung des Stilbendicarbonsäureanhydrids.}

Zur Nitrirung wurde ein Theil der Verbindung in zehn Theile in Eis gekühlte, farblose Salpetersäure vom spec. Gew. 1,52 eingetragen und eine halbe Stunde bei $0^{0}$, dann eine halbe Stunde bei gewöhnlicher Temperatur stehen gelassen. Beim Eingiessen in Wasser scheidet sich die Säure als farblose Masse $a b$, welche nicht einheitlich ist und sich durch Krystallisation nicht zerlegen lässt.

Zur Constitutionsbestimmung wurden $0,6 \mathrm{~g}$ der Säure in verdünnter Natronlauge gelöst und heiss Permanganatlösung zugegeben, bis auf weitere Zugabe von Permanganat keine Einwirkung mehr stattfand. Es wurde dann mit wenig Bisulfit entfärbt, filtrirt und angesäuert. Die erhaltene Nitrobenzoësäure bestand grösstentbeils aus p-Verbindung. Zur Trennuug von der isomeren Substanz wurde die Nitrosäure mit Baryumcarbonat und ziemlich viel Wasser bis zur neutralen Reaction gekocht und heiss filtrirt. Es schieden sich beim Erkalten nur Spuren eines Baryumsalzes ab, welches sich nicht mit Sicherheit als der m-Nitrobenzoësäure angehörig betrachten liess. Das Filtrat lieferte nach dem Eindampfen ein Barytsalz, 
aus dem reine p-Nitrobenzoësäure vom Schmelzp. $238^{\circ}$ in Freiheit gesetzt werden konnte. Die Mutterlauge vom Barytsalz ergab schliesslich ein Gemenge von p- und o-Nitrobenzoësäure, letztere wurde durch die Krystallform (lange Nadeln) und den Geschmack identificirt.

\section{Reduction der nitrirten Stilbendicarbonsäure.}

2,4 g Nitroproduct wurden bei gewöhnlicher Temperatur mit einer Lösung von $10,8 \mathrm{~g}$ Zinnchlorür in der gleichen Menge Wasser und der doppeiten concentrirter Salzsäure mehrere Stunden stehen gelassen; dabei findet allmähliche partielle Lösung und Abscheidung eines Zinndoppelsalzes statt. Es wurde dann auf dem Wasserbade erhitzt, bis eine Veräuderung nicht mehr zu bemerken war. Das ausgeschiedene Zinndoppelsalz (A) wurde von der Flüssigkeit (B) getrenut und beide heiss mit Schwefelwasserstoff behandelt. A lieferte nach dem Filtrireu eine schwach gelbe Lösung, aus der sich nach dem Eindampfen gelbe Krystalle in geringer Menge abschieden. Dieselben lösen sich in organischen Lösungsmitteln nur sehr schwierig, dagegen leicht in verdünnten Säuren, Soda und Natriumacetatlösungen, werden auch von heissem Wasser leicht aufgenommen und scheiden sich nach dem Erkalten in feinen, verwachsenen Nadeln ab. Die Substanz begann sich gegen $130^{\circ} \mathrm{zu}$ färben und zersetzte sich allmählich gegen $180^{\circ}$.

Die entzinnte Lösung B wurde ebenfalls eingedampft, wobei sich zunächst beim Erkalten eine geringe Menge Nitroresp. Azoxyverbindung ausschied (anscheinend ein Gemenge von $0-$ und $m$-Verbindung, welche unter den angewandten Versuchsbedingungen nicht glatt reducirt werdeu); das Filtrat hiervon lieferte auf Zugabe von Natriumacetatlösung p,p-Diamidostilbendicarbonsäure, welche durch ibre Farbe und Eigenschaften mit der beschriebenen Verbindung identificirt wurde. 


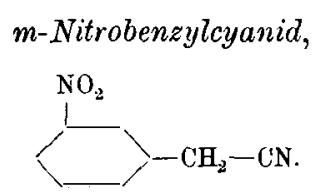

$10 \mathrm{~g} \mathrm{~m}$-Nitrobenzylchlorid wurden mit $45 \mathrm{~g}$ Alkohol und einer Lösung von $2 \mathrm{~g}$ Cyankalium in $4 \mathrm{~g}$ Wasser auf $50-60^{\circ}$ erlitzt. Die Flüssigkeit färbt sich dabei nur schwach dunkel und scheidet Chlorkalium ab. Wenn die Menge desselben nicht mehr zunimmt, was nach $11 / 2-2$ Stunden der Fall ist, versetzt man mit Wasser und verdunstet den Alkohol auf dem Wasserbade, wobei das Reactionsproduct als dunkles Oel zurückbleibt, welches nach einiger Zeit erstarrt. Ausbeute $5 \mathrm{~g}$. Zur Reinigung wurde das Product in Benzol gelöst, auf Zusatz von Ligroïn erfolgt allmählich Krystallisation. Die Verbindung kann indessen so nicht rein erbalten werden. Man muss nunmehr mit kaltem Aether extrabiren, welcher nur das Cyanid aufnimmt und nach dem Concentriren scheidet Ligroïn die reine Substanz ab. Schmelzp. 61-62 .

$0,0666 \mathrm{~g}$ gaben $10,3 \mathrm{ccm}$ Stickgas bei $17,5^{\circ}$ und $736 \mathrm{~mm}$ Druck.

$\begin{array}{ccc} & \text { Berechnet für } & \text { Gefunden } \\ & \mathrm{C}_{8} \mathrm{H}_{8} \mathrm{O}_{2} \mathrm{~N}_{2} & \\ \mathrm{~N} & 17,28 & 17,32\end{array}$

Die Verbindung löst sich leicht in Alkohol, Aether, Benzol, sehr leicht in Aceton und Chloroform, schwer in Ligroïn.

Die von Aether aus dem Rohcyanid nicht gelöste Substanz wird von heissem Alkobol aufgenommen und krystallisirt beim Erkalten in kurzen, verwachsenen Krystalleu vom Schmelzpunkt $149^{\circ}$; welche nicht weiter untersucht wurden. 


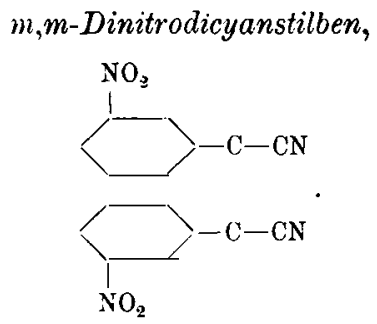

4,8 g m-Nitrobenzylcyanid (es wurde ein einmal krystallisirtes Rohproduct verwandt) wurden in $24 \mathrm{~g}$ wasserfreiem Methylalkohol gelöst, in Eis gekühlt, eine Lösung von $7,7 \mathrm{~g}$ Jod in $25 \mathrm{~g}$ wasserfreiem Aether zugegeben und unter fortwäbrender Kühlung $1,6 \mathrm{~g}$ Natrium in $16 \mathrm{~g}$ Methylalkohol zutropfen gelassen. Schon wäbrend der Reaction scheidet sich das Condensationsproduct krystallinisch aus, ein Farbenumschlag tritt nicht ein. Ausbeute $3,2 \mathrm{~g}$.

Die Substanz ist sehr schwer löslich in heissem Alkohol, Toluol, Chloroform und Eisessig, leichter in heissem Aceton; sie krystallisirt je nach Lösungsmittel in kurzen Stäbchen oder kleinen, sechsseitigen, fast farblosen Plättchen. Schmelzp. $233^{\circ}$ unter Dunkelfärbung.

$0,1791 \mathrm{~g}$ gaben $27,5 \mathrm{ccm}$ Stickgas bei $17^{\circ}$ und $735 \mathrm{~mm}$ Druck.

$\begin{array}{ccc} & \text { Berechnet für } & \text { Gefunden } \\ & \mathrm{C}_{10} \mathrm{H}_{8} \mathrm{~N}_{4} \mathrm{O}_{4} & \\ N & 17,5 & 17,23\end{array}$

m,m-Diamidodicyanstilben.

Die Nitroverbindung wurde in heissem Eisessig gelost und mit der bercchneten Menge Zinnchlorür in concentrirter salzsaurer Lösung versetzt. Bei fortschreitender Reduction färbt sich die Flüssigkeit gelb. Es wurde schliesslich noch etwas Salzsäure zugegeben, worauf sich allmählich das Zinndoppelsalz in farblosen Krystallen absonderte. Dieselben wurden in heissem Wasser suspendirt, mit Natronlauge versetzt und es schied sich dann das orangegelbe Cyanid aus. Dasselbe krystallisirt aus heissem 
Alkohol in gebogeneu, verschlungenen Fäden vom Schmelzpunkt $187^{\circ}$, ist löslich in heissem Benzol, Chloroform, schwer löslich in Ligroïn. Das salzsaure Salz ist farblos und schwer löslich in Wasser.

$0,1103 \mathrm{~g}$ gaben $20,9 \mathrm{ccm}$ Stickgas bei $14^{\circ}$ und $738 \mathrm{~mm}$ Druck.

$$
\begin{array}{ccc} 
& \text { Berechnet fuir } & \text { Gefunden } \\
& \mathcal{C}_{16} \mathrm{H}_{1.5} \mathrm{~N}_{1} & \\
\mathrm{~N} & 21,54 & 21,64
\end{array}
$$

$m, m$-Dinitrostilbendicarbonsäureanhydrid.

$1 \mathrm{~g}$ Cyanid wurde mit $15 \mathrm{~g}$ Eisessig und $11 / 2 \mathrm{~g}$ concentrirter Salzsäure sechs Stunden im Rohre auf $185-190^{\circ}$ erhitzt. Die kaum gefärbte Flüssigkeit schied beim Erkalten Krystalle ab $(0,8 \mathrm{~g})$. Dieselben wurden durch Kochen mit verdünnter Natronlauge in Lösung gebracht, worauf beim Ansäuern das Anbydrid ausfiel. Die Substanz ist schwer löslich in heissem Alkohol, Toluol, ziemlich leicht in heissem Aceton und Eisessig und krystallisirt daraus beim Erkalten, aus letzteren Lösungsmitteln in langen, farblosen Nadeln. Schmelzp. 249-249,5 .

Von Soda wird die Verbindung nur schwierig, erst beim kiochen gelöst.

$0,1409 \mathrm{~g}$ gaben $0,291 \mathrm{CO}_{2}$ und $0,0323 \mathrm{H}_{2} \mathrm{O}$.

$0,1264 \mathrm{~g} \quad, 9,5 \mathrm{ccm}$ Stickgas bei $18,5^{\circ}$ und $737 \mathrm{~mm}$ Druck.

Berechnet fiir Gefunden $\mathrm{C}_{10} \mathrm{H}_{8} \mathrm{O}_{7} \mathrm{~N}_{2}$

$\begin{array}{rrr}\mathrm{C} & 56,47 & \overline{5} 6,33 \\ \mathrm{H} & 2,35 & 2,54 \\ \mathrm{~N} & 8,24 & 8,39\end{array}$

m,m-Azoxystilbendicarbonsäureanhydrid.

In saurer Lösung gelang die Reduction nicht.

1,4 g Dinitrostilbendicarbonsäureanbydrid wurden mit der doppelten Menge Wasser und tropfenweisem Zusatz von Natronlauge in Lösung gebracht, dann $10 \mathrm{~g}$ Ammoniak zugefügt und mit Zinkstaub auf dem Wasserbade erhitzt, wobei die Flüssig- 
keit rein gelbroth wird. Nach dem Filtriren wurde mit Essigsäure versetzt und erwärmt, um die Substanz in den krystallinischen Zustand überzufübren; sie bildet dann ein hellgelbes Pulver. Dasselbe enthält noch Zink, wurde deshalb nochmals mit verdünnter Salzsäure erbitzt, wieder filtrirt und ausgewaschen und war nun aschefrei. Die Substanz war in allen geprüften Lösungsmitteln unlöslich oder schwer löslich, so dass sie nicht krystallisirt werden konnte. Die in Folge dessen nicht ganz scharfen Analysenzahlen lassen keine andere Deutung $z u$ als die oben angenommene. Die Verbindung ist unlöslich in Salzsäure, concentrirte Schwefelsäure löst mit rotbgelber Farbe; von Soda wird sie schon in der Kälte allmählich aufgenommen. Die Substanz bat keinen Schmelzpunkt und färbt sich von $245^{\circ}$ an langsam dunkel und sintert zusammen. Sie liess sich nicht weiter reduciren.

I. $0,1559 \mathrm{~g}$ gaben $0,3819 \mathrm{CO}_{\mathrm{2}}$ und $0,0403 \mathrm{H}_{2} \mathrm{O}$. $0,1501 \mathrm{~g} \quad " \quad 0,3659 \mathrm{CO}_{\mathrm{g}} \quad, \quad 0,0383 \mathrm{H}_{2} \mathrm{O}$.

II. $0,1747 \mathrm{~g}, \quad 15,6 \mathrm{ccm}$ Stickgas bei $17,5^{\circ}$ und $733 \mathrm{~mm}$ Druck.

\begin{tabular}{cccc} 
& Berechnet & \multicolumn{2}{c}{ Gefunden } \\
& $\mathrm{C}_{\mathrm{tv}} \mathrm{H}_{5} \mathrm{O}_{4} \mathrm{~N}_{2}$ & I. & II. \\
$\mathrm{C}$ & 65,75 & 66,80 & 66,48 \\
$\mathrm{H}$ & 2,74 & 2,83 & 2,83 \\
$\mathrm{~N}$ & 9,6 & 9,96 & -
\end{tabular}

- m,m. Diamidostilbendicarbonsäureanhydrid.

m,m-Diamidodicyanstilben wurde in beissem Alkohol gelöst und mit einer concentrirten alkoholischen Lösung von Kali und Natronhydrat so lange erhitzt (vier bis fünf Stunden), bis die rothgelbe Farbe in ein helles Gelb verwandelt war und sich nicht weiter änderte. Es wurde jetzt Wasser zugesetzt, der Alkohol verdampft, nach dem Erkalten filtrirt und mit Essigsäure angesäuert, worauf die Ausscheidung des Reactionsproductes erfolgte. Dasselbe ist in fast allen Lösungsmitteln schwer löslich, löst sich aber in Benzoësäureester, besser noch, in Aethyleabromid und scheidet sich daraus in structurlosen hellrotheu Aggregaten ab, enthält aber dann noch erhebliche 
Mengen Asche. Da die weitere Reinigung mit geringen Mengen Material nicht gelang, wurde die Substanz in diesem Zustande analysirt und die gewogene Asche auch für die N-Bestimmung in Rechnung gezogen.

\begin{tabular}{ccc}
$0,1283 \mathrm{~g}$ gaben $0,3204 \mathrm{CO}_{2}$ und $0,0488 \mathrm{H}_{2} \mathrm{O}$. \\
$0,0919 \mathrm{~g} \quad, \quad 7,8 \mathrm{ccm}$ Stickgas bei $24^{\circ}$ und $741 \mathrm{~mm}$ Druck. \\
\multicolumn{4}{c}{ Berechnet fitr } \\
$\mathrm{C}$ & $\mathrm{C}_{18} \mathrm{H}_{12} \mathrm{O}_{3} \mathrm{~N}_{2}$ & Gefunden \\
$\mathrm{C}$ & 68,57 & 68,11 \\
$\mathrm{H}$ & 4,28 & 4,23 \\
$\mathrm{~N}$ & 10,0 & 10,38
\end{tabular}

o,o-Azoxystilbendicarbonsäureanhydrid.

Wird die feingepulverte 0,0 -Dinitrostilbendicarbonsäure mit salzsaurer Zinnchlorürlösung auf dem Wasserbade erbitzt, so färbt sie sich allmählich gelb, ohne in Lösung zu gehen. Nachdem keine Aenderung mehr zu bemerken war, wurde das Product abfiltrirt und mit Schwefelwasserstoff behandelt, wodurch auch keine Veränderung erfolgte. Die Substanz wurde jetzt mit Wasser und wenig verdünnter Natronlauge in Lösung gebracht; auf Zusatz von concentrirter Lauge schied sich beim Erkalten das Natronsalz in schwach gelben, büschelförmig vereinigten Nadeln ab. Die aus dem Salz in Freiheit gesetzte Säure ist ganz schwach gelb und entspricht in ihren Eigenschaften dem m,m-Azoxystilbendicarbonsäureanhydrid; sie ist unlöslich in Säuren und wird wie jene Verbindung schon von verdünnter Sodalösung allmählich mit hellgelber Farbe aufgenommen. Organische Lösungsmittel sind fast ohne Einwirkung. Bei $270^{\circ}$ ist sie noch unverändert.

$0,0474 \mathrm{~g}$ gaben $4,2 \mathrm{~cm}$ Stickgas bei $26^{\circ}$ und $74 \check{\mathrm{mm}}$ Druck.

$$
\begin{array}{ccc} 
& \text { Berechnet für } & \text { Cefunden } \\
& \mathrm{C}_{\mathbf{1 0}} \mathrm{H}_{5} \mathrm{O}_{4} \mathrm{~N}_{\mathbf{3}} & \\
\mathrm{N} & 9,6 & \mathbf{9 , 6 2}
\end{array}
$$

Dieselbe Substanz resultirte anscheinend, wie nur mit geringen Mengeu Substanz festgestellt werden kounte, beim Kochen der Nitroverbindung mit Ziukstaub und Ammoniak. 
Indess scheint die Reduction weiter zu gehen, wenn man die Nitroverbindung in Eisessiglösung mit Zinnchlorür reducirt. Nach längerem Kochen scheidet sich ein Theil des Zinndoppelsalzes, der Rest auf Zusatz von etwas Wasser ab. Es war aber nicht möglich, die Substanz aschefrei darzustellen; es scheint jedoch das erhaltene Product den Verbindungen aus Dichloressigsäure und Anilin sehr ähnlich zu sein. Berücksichtigt man ferner, dass die beiden isomeren Substanzen (para und meta) in ihrem Verbalten den fraglichen Verbindungen in Bezug auf Schmelzpunkt und Löslichkeitsverhältnisse ausserordentlich gleichen, aber dem Aussehen (roth resp. rothgelb) nach davon verschieden sind, dass ferner in beiden Fällen die Amidocyanide dieselbe Färbung baben wie die Amidosäuren, so spricht auch der Umstand, dass das o-Amidocyanid gelb und auffallend schwer löslich ist, dafür, dass die zugehörige Säure denselben Farbenton und gleiche Eigenschaften besitzt, wie die in Rede stehenden.

Die früber geäusserte Annahme ${ }^{5}$ ), dass das Material leichter beschaff bar werden würde, da das 0 -Nitromandelsäurenitril durch Alkali anscheineod in Di-o-nitrostilbendicarbonsäure verwandelt würde, bestätigte sicb nicht. Es entsteht dabei im Wesentlichen 0 -Nitrosobenzoësäure ${ }^{6}$ ).

\section{Einwirkung von Dichloressigsäure auf m-Toluidin.}

2,6 g Dichloressigsäure (ein Mol.) wurden mit $15 \mathrm{ccm}$ Wasser verdünnt und mit Natronlauge neutralisirt. Nach Zugabe von $3 \mathrm{~g}$ krystallisirtem Natriumacetat und $2,2 \mathrm{~g} \mathrm{~m}$-Toluidin (ein Mol.) wurde funf bis sechs Stunden auf dem Wasserbade erhitzt, wobei sich die Base allmählich unter schwacher Färbung in das Reactionsproduct umwandelt. Die erkaltete Masse wird von der wässrigen Schicht getrennt und mit verdünnter Salzsäure behandelt; dabei geht ein Theil der Sub-

5) Ber. d. deutsch. chem. Ges. 37, 948.

$\left.{ }^{6}\right)$ Ber. d. deutsch. chem. Ges. 39, 2335, 2338. 
stanz mit gelber Farbe in Lösung, während der andere Theil sich in ein rothgelbes Pulver verwandelt. Letzteres ist in den meisten Lösungsmitteln schwer löslich, leichter in heissem Eisessig und Alkohol, obne indessen daraus zu krystallisiren, ferner unlöslich in Soda. Beim Erhitzen färbt sich die Substanz von $200^{\circ}$ an langsam dunkel, ist bei $260^{\circ}$ noch nicht geschmolzen und bläht sich beim höheren Erhitzen langsam auf.

Aus der salzsauren Lösung wird die freie Amidoverbindung durch essigsaures Natron in gelben Flocken ausgefällt, welche sich in Soda leicht lösen. Versuche zur Reinigung beider Substanzen lieferten keine krystallisirten Verbindungen, so dass die weitere Untersuchung unterbleiben musste. Sicher gestellt ist indessen durch das Verbalten der Substanzen, dass dieselben Homologe der Säuren aus Dichloressigsäure und Anilin und das homologe Toluidid sind.

Einwirkung von Dichloressigsäure auf $m$-Xylidin-1,3,4.

(Zum Theil mit Dr. P. Leyden.)

19,5 g Dichloressigsäure wurden mit Kalilauge genau neutralisirt, $21 \mathrm{~g}$ Natriumacetat zugegeben und mit Wasser auf das Gesammtgewicht von $147 \mathrm{~g}$ gebracht; nach Zusatz von $37,2 \mathrm{~g} \mathrm{~m}$-Xylidin wurde 24 Stunden unter öfterem Umschütteln auf dem Wasserbade erbitzt. Die Base batte sich dann in eine hellrothe, körnige Krystallmasse verwandelt. Nach dem Absaugen und Auswaschen mit Wasser wurde die Substanz mit Alkohol verrieben und es hinterblieben nach dem Filtriren $28,8 \mathrm{~g}$ hellgelbe, verfilzte Nadeln. In reinem Zustande ist die Substanz farblos; sie ist aber in diesem Zustande schwer zu erhalten, da sie oberflächlich leicht, wenn auch immer nur in geringer Menge oxydirt wird. Die Neigung zur Oxydation ist lange nicht so gross, wie bei der entsprechenden Verbindung aus p-Toluidin; dort wird in der Regel das Isatinderivat als Hauptreactionsproduct erhalten, wäbrend hier die primäre Substanz bei weitem uberwiegend entsteht. Erschwert wird die Reinigung ferner durch die geringe Löslichkeit in organischen 
Lösungsmitteln. Von siedendem Toluol wird dic Verbindung aufgenommen und krystallisirt dann in schwach fleischfarbenen, sehr feinen Nadelu, welche sich von $220^{\circ}$ ab dunkel färben und gegen $234^{\circ}$ geschmolzen sind. Am bequemsten ist die Reinigung durch fortgesetzte Extraction im Soxhlet'schen Apparate mit Chloroform, wobei sich die Substanz allmählich im unteren Kolben ansammelt.

$0,1529 \mathrm{~g}$ gaben $0,4352 \quad \mathrm{CO}_{2}$ und $0,0964 \mathrm{H}_{2} \mathrm{O}$.

$0,1444 \mathrm{~g} \# 12,4 \mathrm{ccm}$ Stickgas bei $13,5^{\circ}$ und $757 \mathrm{~mm}$ Druck.

Berechnet für

Gefunden $\mathrm{C}_{18} \mathrm{H}_{20} \mathrm{ON}$

$\begin{array}{rrr}\mathrm{C} & 77,14 & 77,62 \\ \mathrm{H} & 7,14 & 7,00 \\ \mathrm{~N} & 10,00 & 10,04\end{array}$

Wegen der oberflächlichen Oxydation der Verbindung felen die Analysenzahlen nicht ganz scharf aus. Die Formel der Substanz ist<smiles>CCC(C)NC1CC(C)CC(C)C1NC(C)C</smiles>

und sie hat die Bezeichnung m-Xylido-2,4-dimethyloxindol.

Sie wird nur von ganz concentrirter Salzsäure gelöst und fällt beim Verdünnen mit Wasser wieder aus. Ammoniakalische Silberlösung wirkt auf die alkoholische Lösung unter Spiegelbildung ein.

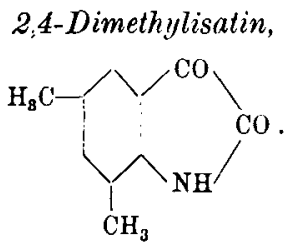

Für die Ueberführung in das zugehörige Isatin erwies sich die Oxydation mit Brom in Eisessig als sehr bequem. $7 \mathrm{~g}$ 
Oxindolkörper wurden mit der zwanzigfachen Menge Eisessig durch Erbitzen gelöst und nach dem Abküblen, wobei die Sub$\operatorname{stanz}$ meist wieder auskrystallisirte, $4 \mathrm{~g}$ Brom (ein Mol.) in $25 \mathrm{~g}$ Eisessig allmählich zugegeben, wobei die Verbindung in Lösung geht; es wurde dann noch eine halbe Stunde auf dem Wasserbade erhitzt. Die anfangs rotbbraune Farbe der Lösung geht dabei über Grun in Hellroth über und die Reaction verläuft in der Weise, dass das Oxindolderivat zunächst bromirt wird, indem ein Bromatom in das Molekül eintritt. Durch Erhitzen erfolgt dann Bromwasserstoffabspaltung unter Bildung des Isatinderivates und darauf Ablösung des Xylidins und Bildung ron Dimetbylisatin.

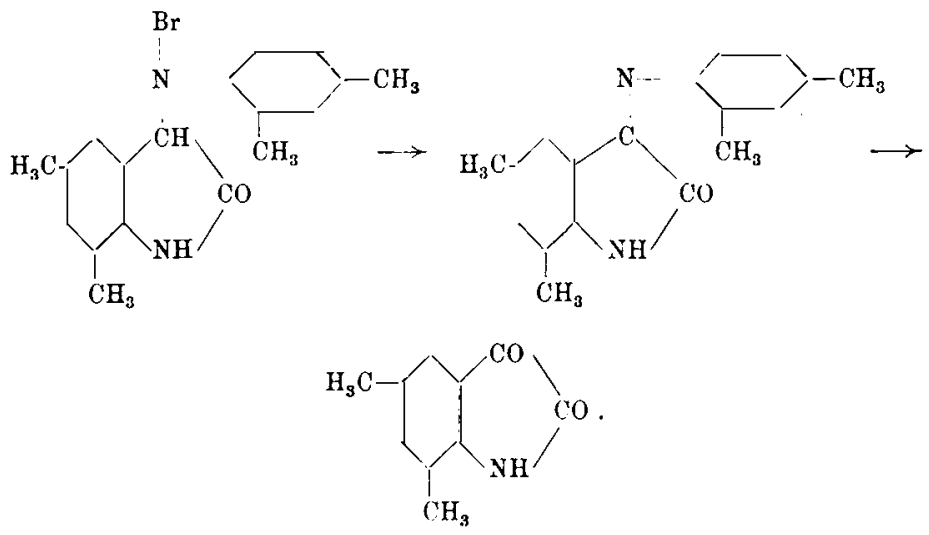

Man verdünnt mit der gleichen Menge Wasser und filtrirt nach längerem Stehen. 2,1 g Ausbeute. Zur Reinigung wird das Product in verdunntem Alkali aufgenommen, wobei die Farbenerscheinung der alkalischen Isatinlösung auftritt und aus dem Filtrate das Dimethylisatin durch Ansäuern als hellrother Niederschlag ausgefällt. Derselbe wird in heisser 50-procentiger Essigsäure gelöst und mit Wasser versetzt, worauf sich die Substanz beim Erkalten in feinen, ausgezackten Blättern und Stäbchen ausscheidet. In beissem Wasser ist sie schwer löslich. Aus heissem Benzol krystallisirt die Verbiudung in gelblichen Nadeln, die aber wohl keine tautomere Form dar- 
stellen, da sie sich in Alkali mit derselben Farbenerscheinung lösen. Die Substanz zeigt die Indopheninreaction und schmilzt bei $243^{\circ}$. Sie ist scbwer löslich in Alkohol und Aether, leicht in Aceton und Cbloroform, kaum löslich in Ligroin.

$0,1761 \mathrm{~g}$ gaben $0,4381 \mathrm{CO}_{2}$ und $0,0756 \mathrm{H}_{2} \mathrm{O}$.

$0,1103 \mathrm{~g}, 8,1 \mathrm{~cm}$ Stickgas bei $14^{\mathrm{p}}$ und $750 \mathrm{~mm}$ Druck.

$\begin{array}{cr}\text { Berechnet für } & \text { Gefunde } \\ \mathrm{C}_{10} \mathrm{H}_{8} \mathrm{O}_{2} \mathrm{~N} & \\ 68,57 & 67,85 \\ 5,14 & 4,77 \\ 8,00 & 8,18\end{array}$

Brom-m-xylido-2,4-dimethyloxindol.

Man kann bei der Bromirung die Reaction auch auf der ersten Stufe festhaiten, wenn man nach Zugabe des Broms, ohne erst zu erwärmen, mit Wasser versetzt, worauf sich alsbald farblose, bromhaltige Nadelu ausscheideu. Zur Entfernung von etwas Oxydationsproduct wird mit Alkohol angerührt und nach dem Absaugen und Trocknen aus Toluol krystallisirt. Die Substanz beginnt sich gegen $185^{\circ} \mathrm{zu}$ färbeu und schmilzt gegen $215^{\circ}$. Sie ist schwer löslich in beissem Aceton, Alkohol und Chloroform.

$0,1754 \mathrm{~g}$ gaben $0,3846 \mathrm{CO}_{3}$ and $0,0797 \mathrm{H}_{2} \mathrm{O}$.

$0,1086 \mathrm{~g}, \quad 10,55 \mathrm{cem}$ Stickgas bei $14,5^{\circ}$ und $758 \mathrm{~mm}$ Druck.

$\begin{array}{cr}\text { Berechnet für } & \text { Gefunde } \\ \mathrm{C}_{18} \mathrm{H}_{28} \mathrm{ON} \mathrm{N}_{2} \mathrm{Br} & \\ 60,17 & 59,80 \\ 5,29 & 5,05 \\ 7,80 & 7,80\end{array}$

2,4-Dimethylisatinphenylhydrazon.

$1 / 2 \mathrm{~g}$ Substanz wurde in 50-procentiger Essigsäure beiss gelöst und Phenylhydrazin zugegeben. Die Flüssigkeit färbt sich gelb und scheidet das Hydrazon in feinen Nadeln ab. Die Substanz ist schwer löslich in heissem Benzol und Ligroun, leichter in Aceton und Chloroform. Sie krystallisirt aus der 
ungefähr 400-fachen Menge absolutem Alkohol beim Erkalten in dünnen, hellgelben Nadeln vom Schmelzp. $272^{\circ}$.

$0,1175 \mathrm{~g}$ gaben $16,5 \mathrm{~cm}$ Stickgas bei $15^{\circ}$ und $757 \mathrm{~mm}$ Druck.

$\begin{array}{ccc} & \text { Berechnet fuir } & \text { Gefunden } \\ & \mathrm{C}_{\mathbf{1 6}} \mathrm{H}_{1 \mathrm{1b}} \mathrm{ON}_{8} & \\ \mathrm{~N} & 15,85 & 16,28\end{array}$

Dichloressigsäure und $m$-Xylidin-1,3,5.

Der Ansatz wurde in denselben Verbältnissen genommen, wie beim m-Xylidin-1,3,4 und die Base in chemisch reinem Zustande verwandt. Die Reaction ging langsam von statten und es schieden sich allmählich schwach gefärbte Krystalle aus. Nach 20 Stunden wurde filtrirt und das Product mit Aether gewaschen. Aus 4,2 g Dichloressigsäure wurden 4,6 g erhalten, welche sich durch ibre Eigenschaften als isomeres Oxindolderivat erwiesen.

\section{3,5-Xylido-3,5-dimethyloxindol.}

Die erhaltene Substanz war einheitlich, erwies sich als schwer löslich in den gebräuchlichen Lösungsmitteln und krystallisirte aus Aethylenbromid in Nadeln oder aus vielem Essigester nach dem Concentriren in kleinen Krystallen. Die Neigung zur Oxydation ist bei mässiger Temperatur geringer als bei den Isomeren; in der Hitze erfolgt die Sauerstoffaufnahme indessen leichter, wie sich daran zeigt, dass die Substanz unter Rothfärbung schmilzt (bei $250^{\circ}$ ) und auch schon vorher von Färbung begleitete Sinterungserscheinungen zeigt.

$0,0839 \mathrm{~g}$ gaben $7,4 \mathrm{ccm}$ Stickgas bei $15,5^{\circ}$ und $750 \mathrm{~mm}$ Druck.

$$
\begin{array}{ccc} 
& \text { Berechnet für } & \text { Gefunden } \\
& \mathrm{C}_{18} \mathrm{H}_{20} \mathrm{ON}_{2} & \\
\mathrm{~N} & 10,0 & 10,17
\end{array}
$$

\section{3,5-Dimethylisatin.}

Die Oxydation des Oxindolderivates gelang nicht mit Brom. Beim Zusammengeben der äquivalenten Menge in Eisessig fand 
zwar partielle Lösung und Ausscheidung des bromhaltigen Derivates statt, aber beim Erwärmen auf dem Wasserbade erfolgte nur geringe Rothfärbung und auch nach längerem Erhitzen fiel beim Verdünnen mit Wasser farblose Substanz aus. Indessen gelang die Bildung der gewünschten Verbindung mittelst Jod.

$1,4 \mathrm{~g}$ Xylidodimethyloxindol wurden in der zwanzigfachen Menge Eisessig gelöst, 1,3 g Jod zugegeben und auf dem Wasserbade erwärmt, wobei allmählich Lösung und Rothfärbung erfolgte. Nach einer halben Stunde wurde mit Wasser verdünnt und auskrystallisiren gelassen. Die abgeschiedene Substanz wurde in Alkohol gelöst und durch Ansäuern des Filtrates in bellgelben Flocken gewonnen. Die Farbenerscheinung der alkalischen Lösung ist die bekannte. Die Verbindung löst sich schwer in heissem Wasser, Benzol, leichter in Aceton und Alkobol und zwar mit gelber Farbe; sie krystallisirt aus beissem Xylol in Nadeln, die zu Zwillingen verwachsen sind oder in Büscheln von gelber Farbe mit geringer Orangenüance und dem Schmelzp. 238--239 . Die Indopheninreaction erfolgt ziemlich langsam, auch geht die Farbe der Schwefelsäure nicht über Grün hinaus.

$0,1095 \mathrm{~g}$ gaben $8,0 \mathrm{ccm}$ Stickgas bei $16,5^{\circ}$ und $748 \mathrm{~mm}$ Druck.

$\begin{array}{ccc} & \text { Berechnet für } & \text { Gefunden } \\ & \mathrm{C}_{10} \mathrm{H}_{8} \mathrm{O}_{2} \mathrm{~N} & \\ \mathrm{~N} & 8,0 & 8,35\end{array}$

\section{3,5-Dimethylisatinphenylhydrazon.}

Die Verbindung wird durch Erhitzen der alkoholischen Lösung des Isatins mit essigsaurem Phenylhydrazin erhalten und scheidet sich schon in der Wärme in Nadeln ab. Sie ist leicht löslich in heissem Benzol, Aceton, Eisessig, Essigäther und krystallisirt aus Alkohol auf Zusatz von Wasser in langen, seideartigen, gelben Nadeln von demselbeu Schmelzp. $238^{\circ}$ bis $239^{\circ}$, den das zu Grunde liegende Dimethylisatin zeigt. 
$0,1064 \mathrm{~g}$ gaben $14,7 \mathrm{ccm}$ Stickgas bei $15,5^{\circ}$ und $755 \mathrm{~mm}$ Druck.

$\begin{array}{ccc} & \text { Berechnet für } & \text { Gefunden } \\ & \mathrm{C}_{16} \mathrm{H}_{15} \mathrm{ON}_{3} & \\ \mathrm{~N} & 15,85 & 16,02\end{array}$

Dichloressigsäure und p-Xylidin.

(Zum Theil mit Dr. P. Leyden.)

Auch dieser Versuch wurde mit denselben Mengenverhältnissen der angewandten Substanzen durcbgeführt. Die Base färbt sich beim Erhitzen des Ansatzes auf dem Wasserbade allmählich dunkler und verdickt sich unter Ausscheidung des Reactionsproductes. Nach Beendigung der Umsetzung wurde die Flusssigkeit abgegossen und der Rückstand mit Alkali aufgenommen, wobei nur Spuren ungelöst blieben, und durch Ausäthern von überschüssiger Base befreit. Auf Zusatz von Essigsäure zur filtrirten Flüssigkeit scheidet sich das Reactionsproduct als hellgelber Niederschlag $a b$, welcher beim Stehen krystallinisch wird. Ausbeute $29 \mathrm{~g}$ aus $19,5 \mathrm{~g}$ Dichloressigsäure. Die Substanz wurde in wenig verdunnter Salzsäure gelöst, wobei $0,93 \mathrm{~g}$ einer gelben Substanz ungelöst blieben. Diese charakterisirte sich durch ihr Verhalten als Ilomologes des Reactionsproductes aus Dichloressigsäure und Anilin; es muss aber in Betracht gezogen werden, dass diese geringe Menge von einer Verunreinigung der angewaudten Base herrühren kann.

Das Filtrat schied auf Zusatz von rauchender Salzsäure ziemlich rasch ein salzsaures Salz aus. Dasselbe wurde mehrmals in der Weise umkrystallisirt, dass in wenig Wasser gelöst und heiss mit dem gleichen Volumen rauchender Säure versetzt wurde. Die völlige Reinigung des Salzes wird etwas beschleunigt, wenn man dasselbe einmal in verdünnter Lösung mit Natriumacetat versetzt, worauf die freie Amidocarbonsäure allmäblich auskrystallisirt, während aus weniger verdünnten Lösungen in der Regel ein basisches Salz sich abscheidet. Hieraus lässt sich dann ein ganz farbloses Salz, welches zwei 
Mol. Salzsäure an ein Mol. Diamido-p-xylylessigsäure gebunden enthält, in Knollen erbalten, die aus feinen Nadeln bestehen.

$0,1794 \mathrm{~g}$, im Vacuum getrocknet, gaben 0,1368 AgCl.

$$
\begin{array}{ccc} 
& \text { Berechnet für } & \text { Gefunden } \\
& \mathrm{C}_{18} \mathrm{H}_{22} \mathrm{O}_{2} \mathrm{~N}_{2} \cdot 2 \mathrm{HCl} & \\
\mathrm{Cl} & 19,12 & 18,87
\end{array}
$$

Die freie Amidocarbonsäure wird auch gewonnen, wenn man die verdünnte wässrige Mineralsalzlösung erhitzt, wobei zunächst Lösung und dann Krystallisation erfolgt. Die Verbindung ist in organischen Solventien sehr schwer löslich und wird am besten durch Umsetzung der sehr verdunnten Salzlösung mit Natriumacetat erhalten, worauf sich die Säure allmählich in feinen, farblosen Krystallkörnern vom Schmelzp. $245^{\circ}$ (Gasentwickelung) abscheidet. Sie hat wahrscheinlich die Formel<smiles>CCC1CC(N)C(C)CC1C(C(=O)O)C1CC(C)NCC1C</smiles>

$0,1869 \mathrm{~g}$ gaben $15,6 \mathrm{~cm}$ Stickgas bei $15,5^{\circ}$ und $738 \mathrm{~mm}$ Druck.

$$
\begin{array}{ccc} 
& \text { Berechnet für } & \text { Gefunden } \\
& \mathrm{C}_{18} \mathrm{H}_{22} \mathrm{O}_{2} \mathrm{~N}_{2} & \\
\mathrm{C} & 9,39 & 9,47
\end{array}
$$

Charakteristisch wegen seiner Schwerlöslichkeit ist das schwefelsaure Salz. Zu seiner Darstellung wurde $1 \mathrm{~g}$ salzsaure Verbindung in der 50-fachen Menge Wasser gelöst und in der Hitze mit überschüssiger Schwefelsäure versetzt, worauf die Verbindung sich in farblosen. Knollen, welche sich aus feinen Nädelchen zusammensetzen, abscheidet. Sie wurde noch warm filtrirt und im Vacuum getrocknet.

$0,1720 \mathrm{~g}$ gaben $0,342 \mathrm{CO}_{2}$ und $0,093 \mathrm{H}_{2} \mathrm{O}$.

$0,1723 \mathrm{~g} \quad, \quad 10, \tilde{\mathrm{c}} \mathrm{cm}$ Stickgas bei $15, \tilde{5}^{0}$ und $762 \mathrm{~mm}$ Druck.

$0,1791 \mathrm{~g}, 0,1094 \mathrm{BaSO}_{4}$. 


$\begin{array}{ccr} & \text { Berechnet für } & \text { Gefunden } \\ & \mathrm{C}_{18} \mathrm{H}_{22} \mathrm{O}_{2} \mathrm{~N}_{2} \cdot \mathrm{H}_{2} \mathrm{SO}_{4} & \\ \mathrm{C} & 54,55 & 54,23 \\ \mathrm{H} & 6,11 & 6,01 \\ \mathrm{~N} & 7,09 & 7,14 \\ \mathrm{~s} & 8,35 & 8,38 \\ & & \\ & \text { Di-benzoylamido-p-xylylessiysäure, } \\ & \mathrm{HO} \mathrm{H}_{2} \mathrm{C}-\mathrm{CH}\left[\mathrm{C}_{6} \mathrm{H}_{2}\left(\mathrm{CH}_{3}\right)_{2} \text { NH.CO. } \mathrm{C}_{6} \mathrm{H}_{5}\right]_{2} \text {. }\end{array}$

Die Verbindung scheidet sich auf Zugabe von Benzoylchlorid zur sodaalkalischen Lösung der Säure krystallisirt $a b$ und zwar wesentlich als freie Säure ${ }^{7}$ ). Sie ist in heissem Aceton und Toluol schwer löslich, leichter in Eisessig und Chloroform und wird aus diesem Lösungsmittel auf Zusatz von Petroläther in mikroskopischen Nädelchen erbalten vom Schmelzpunkt $182-183^{\circ}$.

$0,0705 \mathrm{~g}$ gaben $3,35 \mathrm{ccm}$ Stickgas bei $15,5^{\circ}$ und $771 \mathrm{~mm}$ Druck.

$$
\begin{array}{ccc} 
& \text { Berechnet für } & \text { Gefinden } \\
\mathrm{N} & \mathrm{C}_{32} \mathrm{H}_{30} \mathrm{~N}_{2} \mathrm{O}_{4} & \\
5,53 & & \\
& & \\
& \text { Di-p-xylylessigsäuredisazo- } \beta \text {-naphtol, } \\
& \mathrm{HO}_{2} \mathrm{C}-\mathrm{CH}\left[\mathrm{C}_{6} \mathrm{H}_{2}\left(\mathrm{CH}_{3}\right)_{2} \mathrm{~N}_{2} \cdot \mathrm{C}_{10} \mathrm{H}_{6} \mathrm{OH}\right]_{2} .
\end{array}
$$

Durch Diazotiren von $1 \mathrm{~g}$ salzsaurem Salz mit zwei Mol. Nitrit und Eingiessen in eine Lösung aus $0,87 \mathrm{~g} \not \dot{\beta}$-Naphtol, $2 \mathrm{~g}$ Aetzkali und $20 \mathrm{~g}$ Wasser wurde die Verbindung als scharlachrothes Salz abgeschieden, welches in überschüssigem Alliali schwer löslich ist und erst nach völligem Auswaschen anfing in Lösung zu gehen. Es wurde in viel heissem Wasser gelöst und durch Zusatz von Mincralsäure die freie Azocarbonsäure

7) Diese Thatsache habe ich verschiedentlich beobachtet bei der Benzoylirung von Amidocarbonsäuren, wenn das Natronsalz der benzoylirten Süure, wie im vorljegenden Falle, in überschüssigem Alkali unlöslich ist. Die Unsetzung findet demnach wach folgendem Schema statt :

$$
\mathrm{NH}_{2} \cdot \mathrm{K} \cdot \mathrm{COOH}+\mathrm{C}_{6} \mathrm{H}_{5} \cdot \mathrm{COCl}=\mathrm{C}_{6} \mathrm{H}_{8} \mathrm{CO} \cdot \mathrm{NH}-\mathrm{R}-\mathrm{COOH}+\mathrm{NaCl} \text {. }
$$


erhalten. Sie ist löslich in heissem Toluol und Aceton, etwas schwerer in Alkohol. Man erhält sie in langen, rothen, bronzeglänzenden Nadeln durch I,ösen in warmem Chloroform und Zusatz von Ligroïn bis zur Trübung. Die Verbindung erweicht gegen $195^{\circ}$ und schwillt beim höhereu Erbitzen allmählich an. $0,1592 \mathrm{~g}$ gaben $13,4 \mathrm{~cm}$ Stickgas bei $15,5^{\circ}$ und $736 \mathrm{~mm}$ Druck.

Berechnet für $\mathrm{C}_{38} \mathrm{H}_{32} \mathrm{O}_{4} \mathrm{~N}_{4}$

$N$ 9,21
Gefunden

9,39

\section{Nachschrift.}

Die inzwischen erfolgte Publication von J. Ostromisslensky ${ }^{8}$ ) uber das gleiche Thema ist nicht geeignet, meine Untersuchung in andere Bahnen zu lenken.

Ich will es gar nicht in Abrede stellen, dass es möglich ist, die Umsetzung der Dichloressigsäure mit aromatischen Basen wenigstens theilweise in demselben Sinne $z u$ fuhren, hielt es aber für vortheilhaft, eine Versuchsbedingung zu finden, bei der die Reaction mit den einzelnen Basen möglichst einheitlich verläuft, was mir ja auch gelungen ist; dabei stellte es sich denn heraus, dass die einzelnen Amine in verschiedener Weise reagireu können, wodurch also das geeignete Beobachtungsfeld für die Differenzirung der Reactionen gegeben war. Herr Ostromisslensky hat nuu andere, schon von P. J. Meyer vorgezeichnete Bedingungen gewählt und erhält natürlich auch andere Resultate.

Wie soll man aber das nun verstehen, wenn er sagt, dass nach seinen Untersuchungen die Reaction stets in derselben Richtung (thatsächlich sind nur drei Basen berücksichtigt) vor sich gebt, und im experimentellen Theile angiebt, dass er beim o-Toluidin uur $17 \mathrm{pC}$. und beim Anilin $24 \mathrm{pC}$. der theoretischen Menge des erwarteten Reactionsproductes erhalten hat. That-

8) Ber. d. deutsch. chem. Ges. 40, 4972. 
säcblich sind also bei ihm diese Prozesse uur Nebenreactionen, während die Hauptumsetzungen gar nicht berücksichtigt sind.

Dass ferner die in überwiegender Menge erscheinende Säure aus Dichloressigsäure und 0 -Toluidin nicht die von P. J. Meyer vermuthete Constitution besitzt, ist, wie in dieser Abhandlung erwäbnt ist, auch von mir gefunden, aber aus den dort angegebenen Gründen nicht experimentell behandelt worden. Die Angaben von Ostromisslensky über die erwähnte Verbindung bringen ausser der bereits bekannten Bruttoformel nur Reactionen, welche durch analytische Daten nicht gestützt sind und enthalten deshalb keine genügende Charakterisirung.

Bezüglich des Mechanismus der Umsetzung, die zu den Isatinderivaten führt, ist zu sagen, dass die gegebene Erklärung zwar recht nahe liegt, aber durch nichts bewiesen ist, während ich für meine Auffassung, wie später gezeigt werden soll, gewisse Gründe geltend machen kann. Im Uebrigen sehe ich eben darin die Merkwürdigkeit, dass unter meinen Versuchsbediugungen bei einigen Basen die Oxindolbildung sehr glatt erfolgt, bei anderen dagegen gar nicht oder nur spurenweise, gleichgültig, wie nun dieser Reactionsverlauf ist.

Bemerkt sei ferner, dass die Anmerkung $\left.{ }^{1}\right)$ Seite 4973 natürlich ganz hinfällig ist. Ferner muss es auf Seite 4978 Anmerkung ${ }^{2}$ ) statt „Diese Berichte 38“ heissen: „Diese Annalen 332".

Die weitere Behandlung des Gegenstandes nach den von mir gegebenen Gesichtspunkten bleibt auch ferner vorbehalten. 